\title{
Angelica sinensis polysaccharide promotes the proliferation and osteogenic differentiation of human dental pulp stem cells (hDPSCs) by activating the wnt//-catenin pathway
}

\author{
Tiantian Mao ${ }^{1}$, Youjian Peng ${ }^{1}$, Ruobing Peng ${ }^{1}$, Xiaoying Wei ${ }^{2 *}$ \\ ${ }^{1}$ Department of Stomatology, Renmin Hospital of Wuhan University, Wuhan, China; ${ }^{2}$ Department of Stomatology, Lishui \\ People's Hospital, Lishui, China
}

*Corresponding author: Xiaoying Wei, Department of Stomatology, Lishui People's Hospital, No. 58 Wanfeng Dong Lu, Liandu District, Lishui City, Zhejiang Province, China. Tel: +86-18957093092. Email: weixiaoying11011@163.com

Received: 11 October 2021; Accepted: 27 October 2021; Published: 26 November 2021

(c) 2021 Codon Publications

\section{OPEN ACCESS CC) ORIGINAL ARTICLE}

\begin{abstract}
Human dental pulp stem cells (hDPSCs) are capable of forming mineralized nodules. The proliferation and osteogenic differentiation of hDPSCs are very important for alleviating tooth defects caused by related diseases. Angelica polysaccharide (ASP) is the main bioactive ingredient extracted from the angelica root. ASP has a variety of biological functions, including immune regulation, antitumor activity, and hematopoiesis. However, its possible effects on hDPSCs are still unclear. In this study, we aimed to investigate the role of ASP in periodontal diseases. We found that ASP promoted the proliferation of hDPSCs and osteogenic differentiation of hDPSCs. We further found that it promoted the expression of osteogenic-related genes, including ALP, RUNX2, Col1a1, and OCN. Mechanically, we found that ASP activated the Wnt $/ \beta$-catenin pathway. In conclusion, our results suggested that ASP promoted the proliferation and osteogenic differentiation of hDPSCs via the Wnt/ $\beta$-catenin pathway.
\end{abstract}

Keywords: Angelica polysaccharide; human dental pulp stem cells; osteogenic differentiation; proliferation; Wnt/ $\beta$-catenin pathway

\section{Introduction}

Cell-based tissue engineering is critical in regenerative medicine. Pulp tissues are located in the pulp cavity of teeth. Human dental pulp stem cells (hDPSCs) are capable of forming mineralized nodules (Liu et al., 2021). The cells are fusiform, and can self-renew and multidirectionally differentiate (Luan et al., 2021b). hDPSCs have attracted increasing attention due to their advantages, such as ease of availability, less immune rejection, and avoidance of ethics (Zhou et al., 2019). These excellent functions make hDPSCs suitable sources of tissue repair not only in dentine generation but also in regeneration-related diseases (Zhang et al., 2021; Zhou et al., 2021). Although the research of hDPSCs has made great progress, the study on
hDPSCs is still incomplete. The proliferation and osteogenic differentiation of hDPSCs are very important for alleviating tooth defects caused by related diseases.

The perennial herb Angelica (Oliv. Diels) has been widely used in Asian countries (Nai et al., 2021). Angelica polysaccharide (ASP) is the main bioactive ingredient extracted from the angelica root (Kwon et al., 2021). Most polysaccharides reported in the literature are heteropolysaccharides (Huang et al., 2021). Studies have shown that ASP had a variety of biological functions, including immune regulation, anti-tumor activity, and hematopoiesis (Cheng et al., 2021; Guo et al., 2021). ASP can promote mesenchymal stem cell proliferation and osteoblast differentiation by regulating H19 (Zhu et al., 
2021). It could also contribute to the osteogenic differentiation of hDPSCs and the repair of bone defects in type 2 diabetic rats under high glucose state, which may be related to the activation of the Wnt/ $\beta$-catenin pathway (Song et al., 2021). However, its possible effects on hDPSCs are still unclear.

The Wnt/ $\beta$-catenin pathway is involved in the tooth formation region at every stage of tooth development (Chao et al., 2021). The Wnt/ $\beta$-catenin pathway has been confirmed to play an important role in regulating cell proliferation and differentiation (Luan et al., 2021a; Yang et al., 2021b). Studies have shown that the Wnt/ $\beta$-catenin signaling pathway affected the process of osteogenic or adipogenic differentiation of hDPSCs. In addition, classical Wnt/ $\beta$-catenin significantly promoted apical papilla proliferation and dentin/osteoblast differentiation. Therefore, this pathway could serve as a promising target for the treatment of periodontal diseases.

In this study, we aimed to investigate the role of ASP in periodontal diseases. We confirmed that ASP could promote the proliferation and osteogenic differentiation of hDPSCs via Wnt/ $\beta$-catenin pathway. Our data therefore confirmed that ASP could serve as a promising drug for the treatment of diseases related to dental pulp.

\section{Materials and Methods}

\section{Extraction of ASP}

The raw A. sinensis polysaccharide was extracted from the fresh roots of A sinensis (Oliv.) Diels by boiling water extraction and alcohol precipitation method as previously described (Wang et al., 2016). The refined polysaccharide, named ASP, was obtained by freeze-drying.

\section{Cell culture}

hDPSCs were obtained from the premolars of donors at the age of 12-14 years. All procedures performed in this study involving human participants were in accordance with the standards upheld by the Ethics Committee of Renmin hospital of Wuhan University (Approval no. JZ-1060101) and with those of the 1964 Helsinki Declaration and its later amendments for ethical research involving human subjects. Afterward, the dental pulps were isolated and dispersed with $1 \mathrm{mg} / \mathrm{mL}$ type I collagenase (Gibco, USA) at $37^{\circ} \mathrm{C}$ for $1 \mathrm{~h}$. Then, the tissues were transferred into culturing medium containing low glucose Dulbecco's modified Eagle's medium (DMEM, Gbico, USA), $1 \%$ penicillin-streptomycin, and 10\% fetal bovine serum (FBS, Gibco, USA), and maintained at $37^{\circ} \mathrm{C}$, with the medium changed every 3 days.

\section{CCK-8 assay}

To detect cell viability in hDPSCs, CCK- 8 assay was performed. Briefly, hDPSCs were plated at $3 \times 10^{3}$ cells per well into a 96-well plate and maintained in complete growth media for $24 \mathrm{~h}$. After indicated treatments, cells were treated with $10 \mu \mathrm{l} \mathrm{CCK}-8$ solution at $37^{\circ} \mathrm{C}$ for $1 \mathrm{~h}$. The absorbance of each well was determined with a microplate spectrophotometer at $450 \mathrm{~nm}$ (Bio-Rad Laboratories Inc., Hercules, California, USA).

\section{EdU staining}

After indicated treatment, cells were fixed with $4 \%$ formaldehyde in PBS in each well and permeabilized with $0.5 \%$ Triton X-100 in PBS. Then, Click-iT ${ }^{\circ}$ reaction cocktail was added to each well for $30 \mathrm{~min}$ in dark. Remove the reaction cocktail, then wash each well once with $1 \mathrm{~mL}$ of $3 \%$ BSA in PBS. DAPI was used for nuclear staining.

\section{Alizarin red staining}

After indicated stimulation in hDPSCs, Alizarin red staining (ARS) (HY-120601, MedChemexpress, USA) was performed to evaluate mineral deposition. Cells were fixed with $4 \%$ paraformaldehyde for $15 \mathrm{~min}$ at room temperature, washed thrice with PBS, and stained with $0.5 \%$ ARS. Finally, hDPSCs were incubated with 10\% cetylpyridinium chloride. Then, the absorbance was detected at $560 \mathrm{~nm}$ wavelength via a microplate reader.

\section{Alkaline phosphatase activity}

After centrifuging at $1000 \mathrm{~g}$ for $10 \mathrm{~min}$, cell pellets were sonicated on ice. Cell lysates were collected and kept on ice for subsequent detection. The protein concentration in the supernatant was determined with a BCA kit (Abcam, UK). Reagent 1 and reagent 2 were sequentially added to the lysates and incubated with cells for $15 \mathrm{~min}$ at 37区, and then reagent 3 was added immediately, and vortexed immediately. The OD values of each tube at $520 \mathrm{~nm}$ wavelength were acquired with $0.5 \mathrm{~cm}$ optical path quartz cuvette.

\section{Immunoblot assay}

Proteins were extracted from hDPSCs with RIPA buffer (Beoytime). Then, protein samples were subjected to electrophoresis and transferred onto PVDF membranes. After being blocked with $5 \%$ fat-free milk in TBST buffer for 1 $\mathrm{h}$, the membranes were incubated with primary antibodies targeting RUNX2 (1:1000, Abcam, UK), alkaline phosphatase (ALP) (1:1000, Abcam, UK), OCN (1:1000, CST, USA), Wnt3a (1:1000, Abcam, UK), $\beta$-catenin (1:1000, Abcam, UK), APC (1:1000, Abcam, UK), cyclinD1 (1:1000, Abcam, UK), and GAPDH (1:10000, Abcam, UK) at $4^{\circ} \mathrm{C}$ overnight. Then, the membranes were conjugated 
with indicated secondary antibodies for $1 \mathrm{~h}$. The membranes were developed with ECL kit (Abcam, UK).

\section{Statistical analysis}

Data were displayed as mean \pm SD. Statistical analysis was conducted with GraphPad. Significance was assessed by analysis of variance (ANOVA). P $<0.05$ was considered statistically significant.

\section{Results}

\section{ASP enhances $h D P S C$ s proliferation}

After the dissection of hDPSCs, the effect of ASP on cell viability was assessed. As shown in Figure 1A, ASP treatment with varying concentrations induced enhanced cell viability (Figure 1A). Cell proliferation in response to ASP was detected by EdU staining. ASP treatment significantly elevated cell proliferation in hDPSCs (Figure 1B). These data suggest that ASP enhances proliferation of hDPSCs.

\section{ASP could promote osteogenic differentiation of dental pulp stem cells}

The effect of ASP on hDPSCs osteogenic differentiation was assessed by ALP activity and ASP staining. ALP activity is used as an early marker of osteogenesis in the bone-forming system. It was increased in ASPinduced hDPSCs in a dose-dependent manner (Figure 2A). ARS staining was conducted for the detection of calcium deposition. ASP stimulation induced more calcium deposition at the concentration of $200 \mathrm{ug} / \mathrm{mL}$ (Figure 2B). Thus, these results suggested that ASP promoted osteogenic differentiation of hDPSCs.

\section{ASP promotes osteogenic differentiation in hDPSCs}

To further verify the osteogenic effect of ASP on hDP$\mathrm{SCs}$, marker genes in osteogenesis were detected, including RUNX2, ALP, and OCN. ASP treatment significantly enhanced the level of RUNX2, ALP, and OCN, compared with control cells at the concentration of $100 \mathrm{ug} / \mathrm{mL}$.

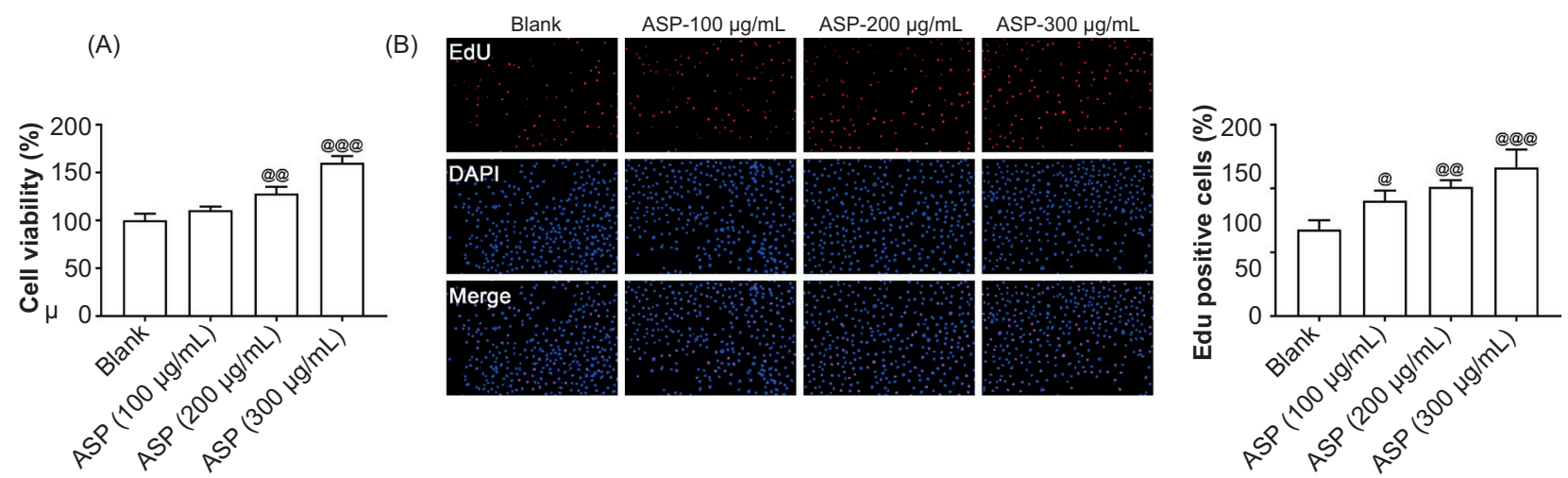

Figure 1. ASP enhances hDPSCs proliferation. (A) Cell viability of hDPSCs treated with increasing concentration of ASP was subjected to CCK-8 assay. (B) EdU staining of hDPSCs treated with increasing concentration of ASP. @, $P<0.05$; @@, $P<0.01$; @@@, $P<0.001$.

(A)

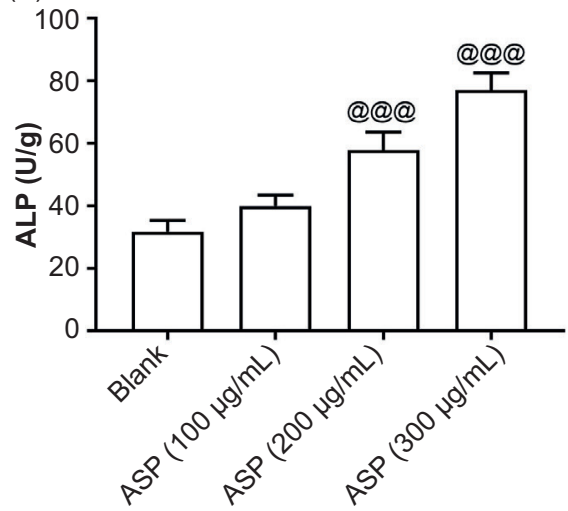

(B)

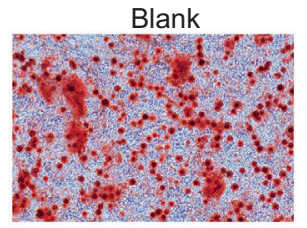

ASP-100 $\mu \mathrm{g} / \mathrm{mL}$

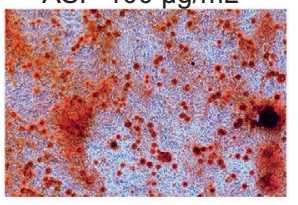

ASP-200 $\mu \mathrm{g} / \mathrm{mL}$

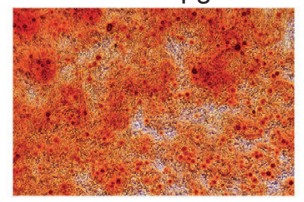

ASP-300 $\mu \mathrm{g} / \mathrm{mL}$

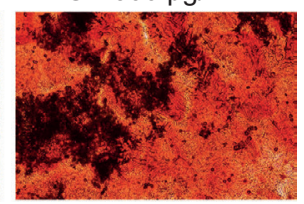

Figure 2. ASP can promote osteogenic differentiation of dental pulp stem cells. (A) Cells treated with ASP were subjected to ALP activity detection. (B) Cells treated with ASP were subjected to calcium deposition detection by ARS. @, $P<0.05$; @@, $P<$ 0.01; @@@, $P<0.001$. 

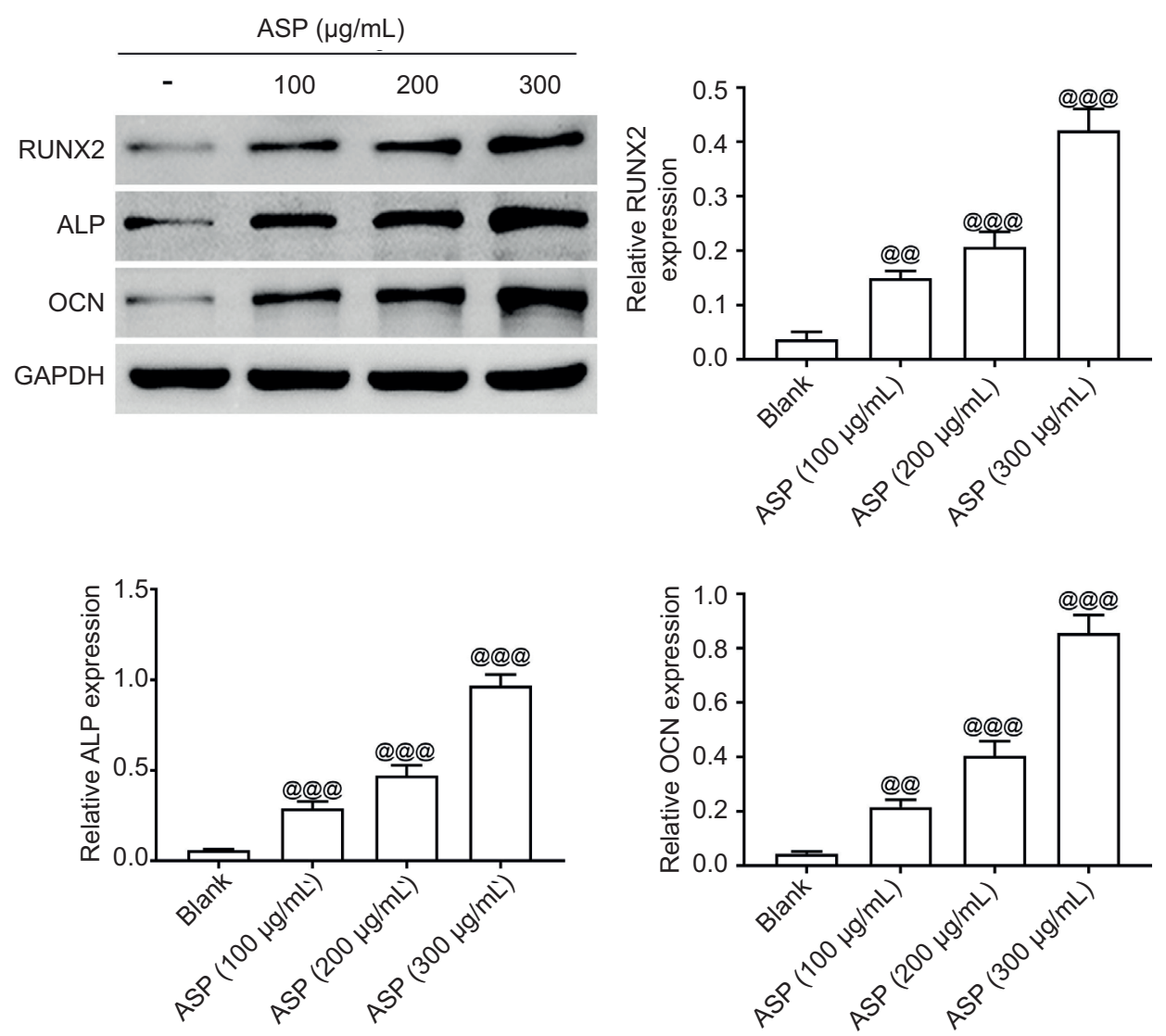

Figure 3. ASP promotes osteogenic differentiation in hDPSCs. The level of RUNX2, ALP, and OCN were detected in cells treated with ASP. @, $P<0.05 ; @ @, P<0.01 ; @ @ @, P<0.001$.

ASP at the concentration of $200 \mathrm{ug} / \mathrm{mL}$ and $300 \mathrm{ug} / \mathrm{mL}$ further enhanced the protein level of RUNX2, ALP, and OCN (Figure 3). Thus, ASP was proved to induce osteogenic differentiation.

\section{ASP promoted the proliferation and osteogenic differentiation of hDPSCs by activating the Wnt/ק-catenin signaling pathway}

To investigate the potential mechanism underlying the promoting of proliferation and osteogenic differentiation of $\mathrm{hDPSCs}$, the Wnt/ $\beta$-catenin signaling pathway was measured. ASP stimulation promoted the level of Wnt3a, $\beta$-catenin, and cyclinD1. However, the level of APC was reduced following ASP treatment at the concentration of $100 \mathrm{ug} /$ $\mathrm{mL}$ (Figure 4). These data implied that ASP promoted the proliferation and osteogenic differentiation of hDPSCs by activating the $\mathrm{Wnt} / \beta$-catenin signaling pathway.

\section{Discussion}

hDPSCs are fusiform and can self-renew and multidirectionally differentiate. In addition, hDPSCs have attracted more and more attention due to the ease of availability, less immune rejection, and avoidance of ethics (Zhou et al., 2021). Therefore, hDPSCs are suitable sources of tissue repair not only in dentine generation but also in regeneration-related diseases, such as diseases related to dental pulp (Liu et al., 2020; Zhou et al., 2019). Recently, cell-based tissue engineering is widely used in regenerative medicine. In this study, we revealed a promising drug, ASP, which has the potential to affect the proliferation and osteogenic differentiation of hDPSCs. Therefore, our results suggested that ASP could serve as a promising drug for the treatment of diseases related to dental pulp.

By Edu and CCK- 8 assays, we noticed that ASP could promote the proliferation of hDPSCs. Furthermore, through ALP and ARS assays, we found that it could contribute to osteogenesis differentiation of hDPSCs. In addition, ASP promoted the expression of osteogenesis genes. Therefore, our results confirmed the key activities of ASP on hDPSCs. The multiple biological activities of ASP in different types of diseases, such as immune regulation, antitumor activity, and hematopoiesis, have been widely revealed (Li et al., 2021). ASP alleviated 

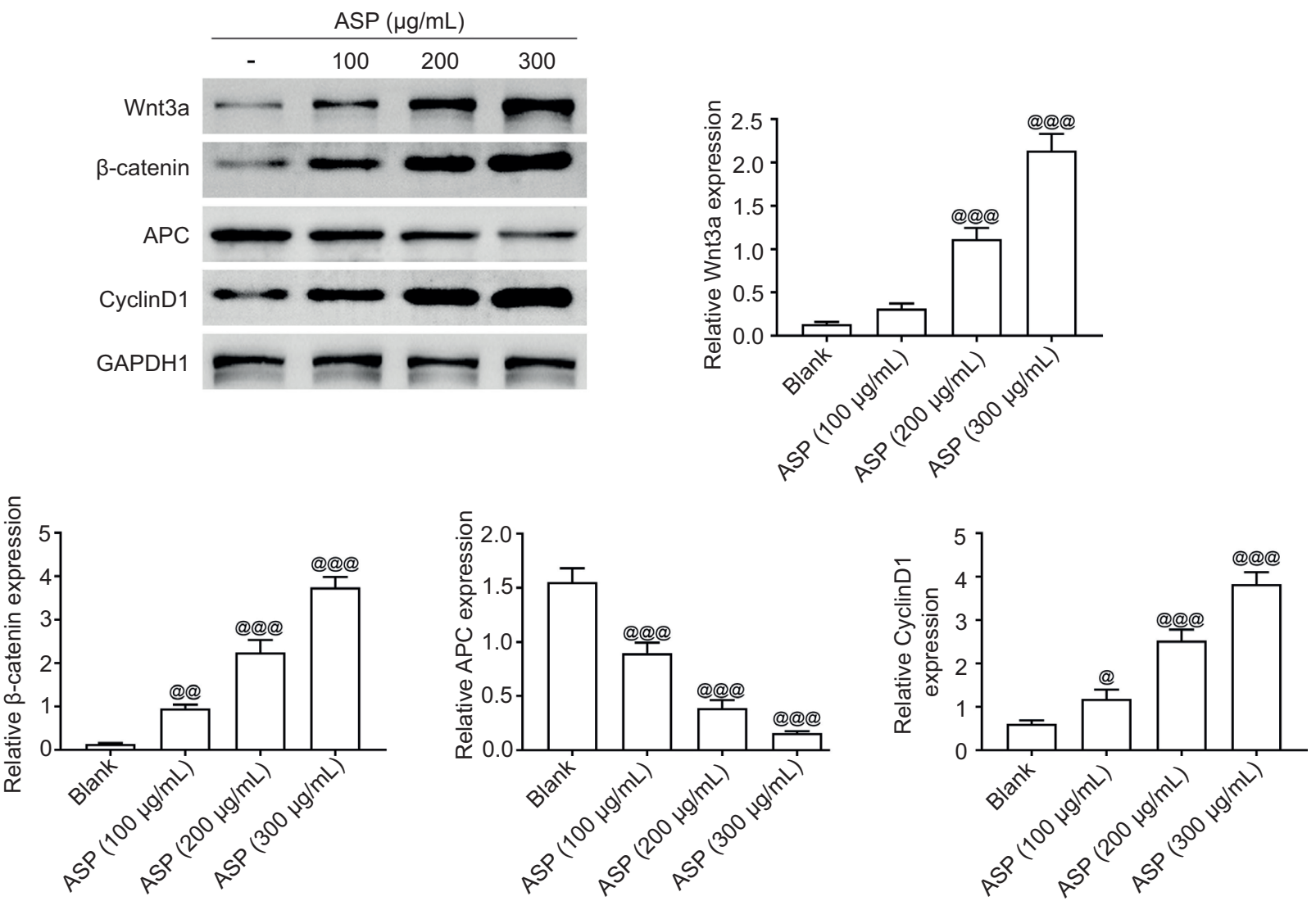

Figure 4. ASP promoted the proliferation and osteogenic differentiation of hDPSCs by activating the Wnt/B-catenin signaling pathway. The level of Wnt3a, $\beta$-catenin, and cyclinD1 were detected in cells treated with ASP. @, $P<0.05 ; @ @, P<0.01$; @@@, $P<0.001$.

myocardial fibrosis and oxidative stress in the heart of hypertensive rats (He et al., 2021). ASP could antagonize 5-FU-induced oxidative stress injury to suppress apoptosis in the liver. ASP attenuated diosbulbin-B-induced hepatotoxicity via the MEK/ERK pathway (Chao et al., 2021). ASP also attenuated SNP-induced apoptosis in osteoarthritis chondrocytes by inducing autophagy ( $\mathrm{Li}$ et al., 2021). In this study, we found its effects on the proliferation and osteogenesis differentiation of hDPSCs. These studies confirmed that ASP played key roles in combating multiple types of diseases (Ali et al., 2021).

The Wnt/ $\beta$-catenin pathway is involved in the progression of tooth development. Multiple proteins and drugs mediated tooth development via this pathway (Chang et al., 2017). For example, BMP9-initiated osteogenic differentiation of tooth germ mesenchymal cells (TGMCS) in mice required Wnt/ $\beta$-catenin activity. Runx2 mediated mouse tooth root development via the activation of Wnt/ $\beta$-catenin pathway (Yang et al., 2021c). Sequential stimulation with BMP4 contributed to the differentiation of human embryonic stem cells (hESCs) into dental epithelium via this pathway. These studies, together with our findings, confirmed that the Wnt/ $\beta$-catenin pathway could serve as a promising target for the treatment of tooth-related diseases.
In addition, the $\mathrm{Wnt} / \beta$-catenin pathway has proved to play an important role in regulating cell proliferation and differentiation. Previous studies showed that the Wnt $/ \beta$-catenin signaling pathway affected the process of osteogenic differentiation of hDPSCs (Su et al., 2020). In addition, $W n t / \beta$-catenin obviously contributed to apical papilla proliferation and dentin/osteoblast differentiation. miR-330-5p derived from plastrum testudinispreconditioned bone MSCs could attenuate oteogenesis via this pathway (Yang et al., 2021a). Overexpression of HOXB4 could promote the protection of hDPSCs against acute lung injury via the activation of the Wnt/ $\beta$ Catenin pathway (Tsuruda et al., 2021). LincRNA also played a vital role in melatonin-mediated osteogenic differentiation of hDPSCs via targeting this pathway. Similarly, we also found that ASP promoted the proliferation and osteogenic differentiation of hDPSCs via this pathway.

In conclusion, we found that ASP promoted the proliferation of hDPSCs and contributed to osteogenic differentiation. Mechanically, we found that ASP activated the $W n t / \beta$-catenin pathway. In conclusion, our results suggested that ASP promoted the proliferation and osteogenic differentiation of hDPSCs via the $\mathrm{Wnt} / \beta$-catenin pathway. 


\section{Acknowledgements}

Not applicable.

\section{Funding}

Not applicable.

\section{Competing interests}

The authors state that there are no conflicts of interest to disclose.

\section{Ethics approval}

Not applicable.

\section{Statement of Human and Animal Rights}

Not applicable.

\section{Statement of Informed Consent}

Not applicable.

\section{Contribution of authors}

Tiantian Mao and Youjian Peng designed the experiments, Ruobing Peng carried out the experiments and analyzed and interpreted the data, and Xiaoying Wei prepared the manuscript with contributions from all co-authors.

\section{References}

Ali, M.Y., Jannat, S., Jung, H.A. and Choi, J.S., 2021. Insulin-mimetic dihydroxanthyletin-type coumarins from angelica decursiva with protein tyrosine phosphatase $1 \mathrm{~B}$ and alpha-glucosidase inhibitory activities and docking studies of their molecular mechanisms. Antioxidants (Basel) 10(2). https://doi. org/10.3390/antiox10020292

Chang, J., Li, Y., Wang, X., Hu, S., Wang, H., Shi, Q., et al. 2017. Polyphyllin I suppresses human osteosarcoma growth by inactivation of Wnt/beta-catenin pathway in vitro and in vivo. Sci Rep 7(1): 7605. https://doi.org/10.1038/s41598-017-07194-9

Chao, Y.H., Yang, W.T., Li, M.C., Yang, F.L. and Lee, R.P., 2021. Angelica dahurica and rheum officinale facilitated diabetic wound healing by elevating vascular endothelial growth factor.
Am J Chin Med 49(6): 1515-1533. https://doi.org/10.1142/ S0192415X21500713

Cheng, C.Y., Huang, H.C., Kao, S.T. and Lee, Y.C., 2021. Angelica sinensis extract promotes neuronal survival by enhancing p38 MAPK-mediated hippocampal neurogenesis and dendritic growth in the chronic phase of transient global cerebral ischemia in rats. J Ethnopharmacol 278: 114301. https://doi.org/10.1016/j. jep.2021.114301

Guo, Y.R., Jin, H., Kim, M., Shin, M.B., Lee, J.H., Maeng, S., et al. 2021. Synergistic neuroprotective effects of mature silkworm and angelica gigas against scopolamine-induced mild cognitive impairment in mice and H2O2-induced cell death in HT22 mouse hippocampal neuronal cells. J Med Food 24(5): 505-516. https://doi.org/10.1089/jmf.2020.4839

He, Y., Zhong, Y., Bao, Z., Wang, W., Xu, X., Gai, Y., et al. 2021 Evaluation of angelica decursiva reference genes under various stimuli for RT-qPCR data normalization. Sci Rep 11(1): 18993. https://doi.org/10.1038/s41598-021-98434-6

Huang, W.Y., Youk, J.S., Han, B.K., Heo, W., Yun, B.S., Kim, J.S.,et al. 201. Improvement of fatigue symptoms and endurance capacity by the combined administration of Cervus elaphus L., Angelica gigas Nakai, and Astragalus membranaceus Bunge. J Med Food 24(6): 577-585. https://doi.org/10.1089/jmf.2020.4743

Kwon, D.A., Kim, Y.S., Kim, S.K., Baek, S.H., Kim, H.K. and Lee, H.S., 2021. Antioxidant and antifatigue effect of a standardized fraction (HemoHIM) from Angelica gigas, Cnidium officinale, and Paeonia lactiflora. Pharm Biol 59(1): 391-400. https:// doi.org/10.1080/13880209.2021.1900878

Li, C., Liu, S., Zheng, J. and Xue, Y., 2021. Angelica sinensis polysaccharide (ASP) attenuates diosbulbin-B (DB)-induced hepatotoxicity through activating the MEK/ERK pathway. Bioengineered 12(1): 3516-3524. https://doi.org/10.1080/21655979.2021.1950280

Liu, S., Wang, Y.N., Ma, B., Shao, J., Liu, H. and Ge, S., 2021. Gingipainresponsive thermosensitive hydrogel loaded with SDF-1 facilitates in situ periodontal tissue regeneration. ACS Appl Mater Interfaces 13(31): 36880-36893. https://doi.org/10.1021/acsami.1c08855

Liu, Y., Fang, J., Zhang, Q., Zhang, X., Cao, Y., Chen, W., et al., 2020. Wnt10b-overexpressing umbilical cord mesenchymal stem cells promote critical size rat calvarial defect healing by enhanced osteogenesis and VEGF-mediated angiogenesis. J Orthop Translat 23: 29-37. https://doi.org/10.1016/j.jot.2020.02.009

Luan, Y., Luan, Y., Feng, Q., Chen, X., Ren, K.-D. and Yang, Y., 2021a. Emerging role of mitophagy in the heart: therapeutic potentials to modulate mitophagy in cardiac diseases. Oxidative Medicine and Cellular Longevity 2021: 13. https://doi. org/10.1155/2021/3259963

Luan, Y., Luan, Y., Yuan, R.X., Feng, Q., Chen, X. and Yang, Y., $2021 \mathrm{~b}$. Structure and function of mitochondria-associated endoplasmic reticulum membranes (MAMs) and their role in cardiovascular diseases. Oxid Med Cell Longev 2021: 4578809. https://doi. org/10.1155/2021/4578809

Nai, J., Zhang, C., Shao, H., Li, B., Li, H., Gao, L., et al. 2021. Extraction, structure, pharmacological activities and drug carrier applications of Angelica sinensis polysaccharide. Int J Biol Macromol 183: 2337-2353. https://doi.org/10.1016/j. ijbiomac.2021.05.213 
Song, X., Kong, J., Song, J., Pan, R. and Wang, L., 2021. Angelica sinensis polysaccharide alleviates myocardial fibrosis and oxidative stress in the heart of hypertensive rats. Comput Math Methods Med 2021: 6710006. https://doi.org/10.1155/2021/6710006

Su, G., Yan, Z. and Deng, M., 2020. Sevoflurane inhibits proliferation, invasion, but enhances apoptosis of lung cancer cells by Wnt/beta-catenin signaling via regulating lncRNA PCAT6/miR326 axis. Open Life Sci 15: 159-172. https://doi.org/10.1515/ biol-2020-0017

Tsuruda, M., Morino-Koga, S., Ogawa, Minetar., 2021. Bone morphogenetic protein 4 differently promotes distinct VE-cadherin precursor stages during the definitive hematopoietic development from embryonic stem cell-derived mesodermal cells. Exp Hematol. https://doi.org/10.1016/j.exphem.2021.08.008.

Wang, K., Song, Z., Wang, H., Li, Q., Cui, Z. and Zhang, Y., 2016. Angelica sinensis polysaccharide attenuates concanavalin A-induced liver injury in mice. Int Immunopharmacol 31: 140148. https://doi.org/10.1016/j.intimp.2015.12.021

Yang, Y., Yang, L., Wu, Y. and Yuan, J., 2021a. [Dexmedetomidinemediated Wnt pathway inhibits sevoflurane-induced cognitive impairment in neonatal rats]. Zhongguo Yi Xue Ke Xue Yuan Xue Bao 43(2): 235-246. https://doi.org/10.3881/j. issn.1000-503X.12913

Yang, Y., Yang, L., Yuan R.-X. and Luan, Y., 2021b. Histone methylation related therapeutic challenge in cardiovascular diseases. Frontiers in Cardiovascular Medicine 8:710053. https://doi. org/10.3389/fcvm.2021.710053
Yang, Z., Liu, J., Fu, J., Li, S., Chai, Z. and Sun, Y., 2021c. Associations between WNT signaling pathway-related gene polymorphisms and risks of osteoporosis development in Chinese postmenopausal women: a case-control study. Climacteric 1-7. https:// doi.org/10.1080/13697137.2021.1941848

Zhang, H., Li, X., Li, J., Zhong, L. and Chen, X., 2021. Chen S. SDF-1 mediates mesenchymal stem cell recruitment and migration via the SDF-1/CXCR4 axis in bone defect. J Bone Miner Metab 39(2): 126-138. https://doi.org/10.1007/s00774-020-01122-0

Zhou, J., Gao, Y.H., Zhu, B.Y., He, W.F., Wang, G., Xian, C.J., et al. 2021. The frequency window effect of sinusoidal electromagnetic fields in promoting osteogenic differentiation and bone formation involves extension of osteoblastic primary cilia and activation of protein kinase A. Cell Biol Int. 45: 1685-1697. https://doi.org/10.1002/cbin.11606

Zhou, J., Gao, Y.H., Zhu, B.Y., Shao, J.L., Ma, H.P., Xian, C.J., et al. 2019. Sinusoidal electromagnetic fields increase peak bone mass in rats by activating Wnt10b/beta-Catenin in primary cilia of osteoblasts. J Bone Miner Res 34(7): 1336-1351. https://doi. org $/ 10.1002 / j b m r .3704$

Zhu, H., You, J., Wen, Y., Jia, L., Gao, F., Ganesan, K. and Chen, J., 2021. Tumorigenic risk of Angelica sinensis on ER-positive breast cancer growth through ER-induced stemness in vitro and in vivo. J Ethnopharmacol 280: 114415. https://doi.org/10.1016/j. jep.2021.114415 